\title{
A PROPOSED NOVEL ARCHITECTURE OF EC CONTROL SYSTEM USING IEEE 802.11n NETWORK AT ITER-INDIA GYROTRON TEST FACILITY
}

\author{
Deepak Mandge1, Nagendra P. Gajjar², Vipal Rathod ${ }^{3}$, Ronak Shah ${ }^{4}$, Rajvi Parmar ${ }^{5}$ and S. Laxmikanth Rao ${ }^{6}$ \\ 1,3,4,5,6 Electron Cyclotron Heating and Current Drive Group in ITER-India, Institute for Plasma Research, India \\ ${ }^{2}$ Department of Electronics and Communication Engineering, Institute of Technology, Nirma University, India
}

\begin{abstract}
IEEE 802.11 Wi-Fi networks are increasingly becoming popular for its use in industrial applications. With the availability of recent amendments to IEEE 802.11 series of standards, particularly IEEE $802.11 \mathrm{n}$, the adoption of Wi-Fi networks for process automation is gaining more focus and importance. The installation of Wireless networks naturally provides reduction in cable and its maintenance related costs, provides increased flexibility and mobility to enhance performance of industrial control system. The IEEE 802.11n supports parameterization that can be set for particular industrial applications and hence it has addressed to the aspects of timeliness and criticality to some extent. This paper proposes the use of IEEE 802.11n network to interconnect field instruments with Siemens PLC controller in harsh EMI/EMC environment. An application example is shown where the alternate control system architecture is developed in which non-critical and non-safety signals are communicated over Wi-Fi. While, for critical and safety signals, traditional hardwired signals methods can be implemented.
\end{abstract}

Keywords:

PLC, Wireless Neworks, IEEE 802.11n, Industrial Communication, Gyrotron, ECH\&CD, Control System

\section{INTRODUCTION}

Wireless communication for industrial automation applications has been the main focus of research in recent years because of great advantages such as increased mobility, easier deployment and configuration, reduced maintenance cost and increased flexibility. With the advent of technology, many industrial standard networks and protocols for wireless networks are available now-a-days. With many more wireless networking technology (such as IEEE 802.11 Wi-Fi, ZigBee, Wireless HART, ISA100) being readily available in the market, one is at an advantage to choose and implement particular technology to suit the industrial application requirements. Wireless technologies are getting widely used in various industry domain for equipment condition monitoring, process measurement and other applications.

The earlier version of IEEE 802.11 [1] standards used Carrier Sense Multiple Access with Collision Avoidance (CSMA/CA) techniques to access the medium. This technique is sufficient for general purpose communication system but not for industrial communication where tight constraints on timely delivery of message, and reliability are utmost important as explained in [2]. Many studies [3]-[6] have been carried out with resepect to appilcability of IEEE $802.11 \mathrm{n}$ networks in industrial envinronment where authors have discussed the advantages of this network with respect to time criticality and reliability, much needed for industrial applications.

The amendments of IEEE 802.11n standard has addressed to some extent the importance of time criticality and reliability in industrial communication system with higher throghput. IEEE 802.11n includes many improvements at both MAC and PHY layer that may bring improvements in performance of Wi-Fi in industrial communication system.

In this paper, a new alternate control system architecture is presented where traditional Ethernet communication is replaced by IEEE $802.11 \mathrm{n} \mathrm{Wi-Fi} \mathrm{links} \mathrm{for} \mathrm{non-safety} \mathrm{and} \mathrm{non-critical}$ signals. The example shown here is of the Electron Cyclotron control system (ECCS) of ITER-India Gyrotron Test Facility (IIGTF) at Ahmedabad. This paper proposes new design architecture of ECCS based on Industrial wireless communication framework where field devices and power supplies are controlled and monitored via Siemens PLC Controller though reliable, secure and dedicated wireless network across IIGTF Lab.

This paper is organized as follows: Section 1 presents overview of wireless networks and particularly applicability of IEEE 802.11n networks. Section 2 discusses overview of ITERIndia Gyrotron Test facility where such networks can be deployed and configured. Section 3 discusses existing architecture of ECCS at IIGTF. Sectin 4 discusses applicability of IEEE $802.11 \mathrm{n}$ network to ECCS. Section 5 presents new proposed architecture of ECCS using Wi-Fi network. Section 6 discusses the design considerations for Wi-Fi network for ECCS and physical protection of equipement. Section 7 outlines the current status and results. Section 8 outlines the conclusion.

\section{ITER-INDIA GYROTRON TEST FACILITY}

The ITER-India has planned to setup an ITER-India Gyrotron Test Facility (IIGTF) at IPR campus for establishing the integrated High power Gyrotron system [7]. The Gyrotron requires High voltage power supplies (HVDC), set of auxiliary power supplies and auxiliary services for its operation. The HVDC includes Main High Voltage Power Supplies (MHVPS, -55KV at 110A) and Body Power Supply (BPS, $35 \mathrm{kV}$ at $100 \mathrm{~mA}$ ). The auxiliary power supplies include Filament power supply (FPS), Vacuum-ion pump power supply (IPS), Super conducting magnet power supply (SCMPS), Collector coil power supplies (CCPS-DC and CCPSsweeping), and Gun coil power supply (GCPS). 


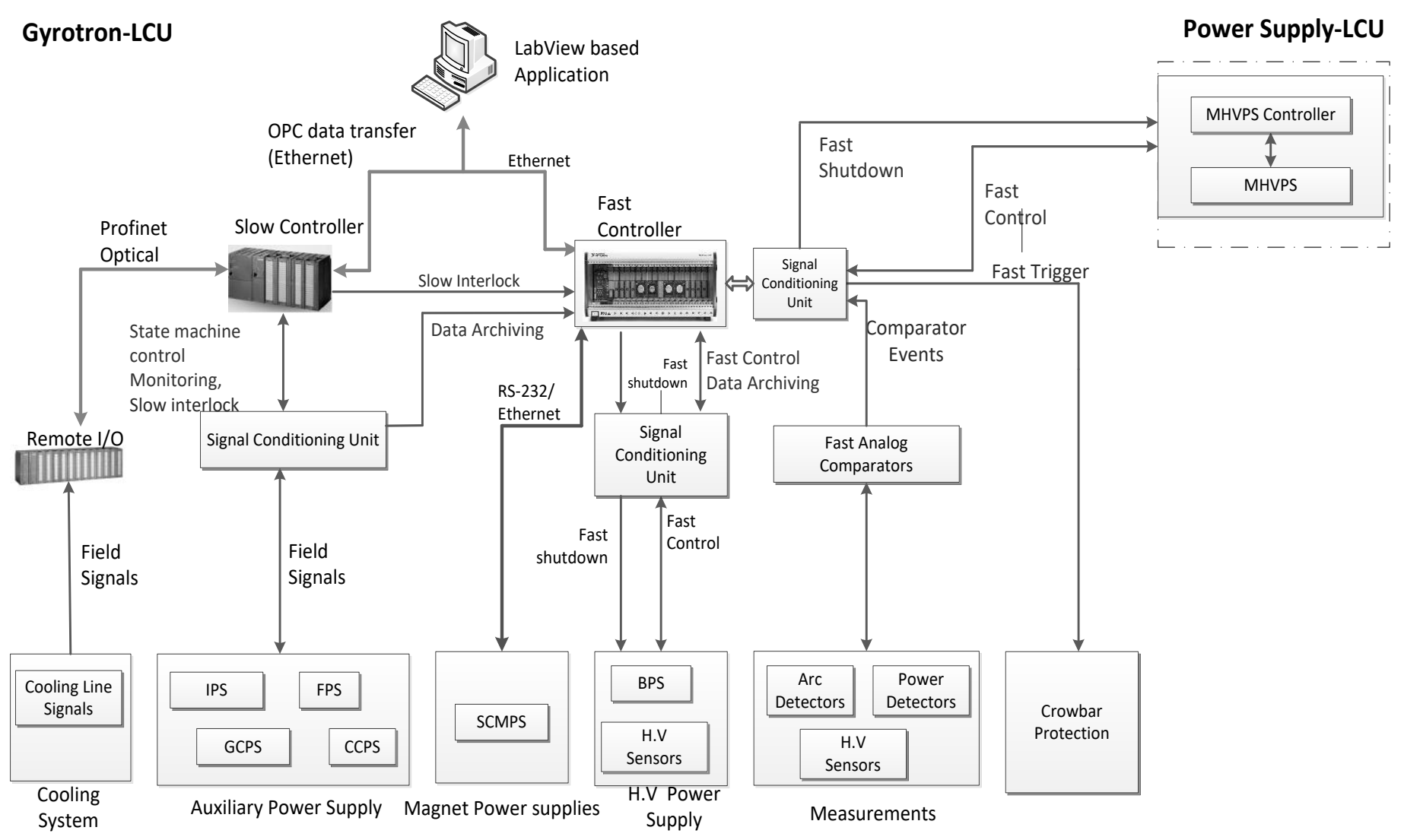

Fig.1. Architecture of ECCS at IIGTF

All these power supplies must be turned ON and OFF in predefined sequence under the supervision of ECCS to operate Gyrotron system safely. The main functions of ECS are sequence control of power supplies, local interlock protection and real time data acquisition of important parameters [8]. The fiber optic transmission based signal conditioning unit (SCU) is required to interface field signals to main EC controllers [9].

\section{PRESENT ECRH CONTROL SYSTEM AT IIGTF (ECCS)}

The present ECCS is used to provide control, monitoring, various alarm handling, data visualization and data storage for Gyrotron system. The ECCS controls and monitors various auxiliary power supplies and auxiliary services (such as cooling systems, vacuum system) connected to Gyrotron System [8]. The main functions of ECCS are given in Table.1.

The control and monitoring function is classified as Auxiliary (Slow) control and Power (Fast) control. Slow control function is implemented for all auxiliary power supplies and controlled through slow controller. Power control will be exerted by fast controller on both high voltage power supplies.

For the data analysis of experimental data, slow data archiving in the frequency range of $1 \mathrm{~Hz}$ to $10 \mathrm{~Hz}$ will be done by slow controller for cooling instrumentation signals. While fast data archiving will be done in the frequency range of $1 \mathrm{kHz}$ to $20 \mathrm{kHz}$ for all power supplies and interlock signals.
Table.1. Main Functions of ECCS

\begin{tabular}{|c|c|}
\hline Main Functions & Descriptions \\
\hline Control and Monitoring & $\begin{array}{l}\text { Auxiliary control (Slow) } \\
\text { Power Control (Fast) }\end{array}$ \\
\hline Data Archiving & $\begin{array}{l}\text { Slow Data Archiving } \\
\text { Fast Data Archiving } \\
\text { Latching of Fast Interlock Events }\end{array}$ \\
\hline Local Protections & $\begin{array}{l}\text { Slow Interlock }(>100 \mathrm{~ms}) \\
\text { Fast Interlock }(<10 \mu \mathrm{s} \&<100 \mathrm{~ms})\end{array}$ \\
\hline Signal Conditioning & $\begin{array}{l}\text { H.V Isolation and Grounding, } \\
\text { Noise Free Transmission }\end{array}$ \\
\hline
\end{tabular}

This fast data archiving will be done by fast controller. Fast interlock events of will be latched at a time scale of $\sim 100 \mathrm{~ns}$ to differentiate between various interlock events.

Local Interlock functions will be implemented both in slow and fast controller. Slow controller will implement local protection function in software and will be performed in slow time scale (>100ms). While fast controller will perform fast interlock functions in the time scale of $<10 \mu \mathrm{s} \&<100 \mathrm{~ms}$.

The use of fiber optic Signal Conditioning unit ensures high voltage and ground isolation for control room equipment. Use of fiber optic cables also ensures noise free transmission of control and monitoring signals. 


\subsection{BRIEF OVERVIEW OF ARCHITECTURE OF ECCS}

The existing architecture of ECCS is show in Fig.1. LabVIEW ${ }^{\mathrm{TM}}$ based Windows station is used as a Local system host for Human machine Interface (HMI), Alarm and archive functions. NI OPC server will be used to access Siemens ${ }^{\circledR}$ PLC (S7-300 System) process variables to develop common HMI in LabVIEW.

The PXIe system will be used as fast controller which consists of RT Controller, DAQ I/O modules, Timing module and FPGA modules. All the systems will communicate over the same Ethernet network for exchange of process variables. Cooling instrumentation signals (Pressure, flow and Temperature signals from various sensors) are connected to remote expansion module of S7-300 PLC and connected to main PLC controller via optical PROFINET. These cooling signals shall be archived in LabVIEW ${ }^{\text {TM }}$ host PC through PLC. Both HV power supplies, power and arc detectors are connected to fast controller via SCU.

All the auxiliary power supplies shall be controlled and monitored by Siemens ${ }^{\circledR}$ PLC via SCU. Fast controller triggers crowbar system in case of detection of fault condition in any of the subsystem of Gyrotron within fast time scale. Fast analog comparators modules are used to detect the events of overvoltage and over current and trigger the crowbar system in order to protect the Gyrotron. All mechanical systems like, vacuum pump, vacuum gauge, chiller, super conducting magnets compressor shall be operated by PLC by Profibus [10] protocol.

Siemens S7-300 PLC System consists of CPU 317-2 PN/DP, SM $321 \&$ SM 322 digital modules, SM-331 \& SM 332 analog modules and ET-200M remote expansion modules IM 153-4. While PXIe system consists of NI PXIe-1082 chassis with 8 slots, PXIe-8133 RT Controller, two digitizer modules (PXI 6259 and PXIe 6368) and PXIe-7854R FPGA module [8].IEEE 802.11n Wi-Fi for ECCS.

Even though Wi-Fi network is majorly considered as an consumer oriented network, it has higher potential to be adopted as an industrial network compared to other wireless technologies such as mobile/cellular network, Bluetooth, ZigBee [11], WirelessHART [12][13][14], etc. In this proposal, IEEE 802.11 Wi-Fi network has been chosen for the implementation for ECCS considering following advantages:

- Higher bandwidth with optimum range

- Lower implementation and maintenance cost

- Supports from majority of vendors,

- Proven security and reliability

- Easy Future expandability and upgradibility

Zigbee (IEEE 802.15.4 standard) is low cost, low power wireless technology for short range communication but it offers very low throughput and low network bandwidth. WirelessHART is first open wireless standard mainly for industrial process control application. Eventhough it is very secure and relible technology, challenges are huge such as time management, mesh networking, low bandwidth and network management [15].

To the other side, there are certain disadvantages that has to be addressed regarding the harsh environment conditions prevailing in industrial application of Wi-Fi networks. Following are the shortcoming of earlier Wi-Fi network to be addressed for industrial application

- Poor network design which results in frequency channel being overloaded, co-channel interference, non-Wi-Fi interference,

- Large metal objects, commonly found in industry, sometimes severly deteriorates and blocks communication.

\subsection{IMPORTANT FEATURES OF IEEE 802.11N}

The various parameters and features of IEEE 802.11n are explained in [3]. After detail simulation and analysis, Author recommended the optimum tuning of IEEE 802.11n parameters for industrial communication, which are given below:

- Use of $40 \mathrm{MHz}$ channel for wider bandwidth

- Use of Greenfield preamble in case no backward compatibility is required

- Use of LDPC (Low density parity Check) code for low SNR environment

- Removal of Short Guard Interval as it may cause inter symbol interference.

- Use of multi antenna $2 \times 2$ STBC (Space Time Block Coding) system with MCS 7 with code rate 5/6.

This parameter settings enhances the IEEE 802.11n network performance specifically in harsh EMC/EMI environment.

\section{PROPOSED ARCHITECTURE OF ECCS}

In this alternate architecture, slow controller communication with field devices and power supplies are implemented by IEEE 802.11n network. The Siemens ${ }^{\circledR}$ PLC system is connected to Industrial graded $\mathrm{Wi}-\mathrm{Fi}$ access point (AP1) in control room which communicates with other $\mathrm{Wi}-\mathrm{Fi}$ clients in the field area attached to different subsystems and auxiliary power supplies. This AP1 shall be configured as per the configuration for IEEE 802.11n explained in section 4 .

The various sensor signals from cooling manifold will terminate at remote expansion module (ET-200M) of Siemens ${ }^{\circledR}$ S7-300 PLC and remote expansion module will transmit these signals over $\mathrm{Wi}-\mathrm{Fi}$ to the main PLC controller. The Wi-Fi router (CL1) connected to remote expansion modules shall be configured to operate in bridge mode and connected to AP1 over IEEE 802.11n configuration.

All the auxiliary power supplies supports legacy serial communication protocol such as RS232 or RS485. So industrial grade serial to Ethernet converter modules shall be installed in metal housing at each power supply modules. These converters converts serial data into Ethernet packets and passed onto CL1. All these converter modules shall be assigned different static IP address. Also, Arc detector device is connected to serial to Ethernet converter for its control and configuration and output signal from the Arc detector is routed to PXIe system via SCU.

The hardwired output signals from the auxiliary power supplies are data acquisitions signals that are routed to PXIe system via SCU. While other signals such as local protection and safety signals are kept hardwired only and are connected to PXIe system via SCU. Hence, at initial phase, only configuration and 
control signals of low response times and low bandwidth are configured to be routed though IEEE 802.11n Wi-Fi network.

\section{SPECIFIC REQUIREMENTS OF WI-FI ACCESS POINTS AND CLIENTS IN IIGTF}

In IIGTF Lab area, Gyrotron shall be installed and operated via high votlage power supplies installed nearby to IIGTF. The magnetic field strengh shall also be higher surrounding to Gyrotron area. Although extreme termperatures and moistures are not expected in IIGTF, power fluctuations caused by nearby HVDC power supply operation, fast switching of Ignitron based crowbar system during Gyrotron operation shall certainly degrade the performance of $\mathrm{Wi}-\mathrm{Fi}$ equipment(AP1 and CL1). The induction motor and its drives used for water cooling system can also cause discontinuous currents and electromagnetic interference for Wi-Fi equipment. Also, the effect of X-ray radiation produced by Gyrotron collector needs to eliminated to safeguard the Wi-Fi equipment.

Hence, proper physical protection for Wi-Fi clients and access points and optimum design of network are key factors that needs to be worked out based on above mentioned sources of disturbances.

\subsection{DESIGN CONSIDERATIONS FOR NETWORK CONFIGURATION}

Following are the major points to be considered while designing the network:

- Wi-Fi access point AP1 and CL1 must be configured for $40 \mathrm{MHz}$ channel bandwidth, with Greenfield preamble for faster communication, with LDPC code for harsh environment, use of double antenna resulting in 2 X 2 STBC with MCS 7 configuration of IEEE 801.11n parameters. needs to be paid towards channel planning to avoid channel congestion [16]. As the utility Wi-Fi network is also installed nearby, the frequency of utility Wi-Fi router should be a separate from the frequency of Wi-Fi device AP1.

- As there is only one Wi-Fi access point AP1 and client CL1 for ECS, there will not be any requirement of smooth roaming and faster handover time from one access point to another point.

- Wi-Fi AP1 and its antenna shall be mounted at efficient positioning to avoid communication blockage due to large metal sheet or metal object which can result in risk of communication failure [16].

\subsection{DESIGN CONSIDERATION PHYSICAL PROTECTION}

To ensure that a wireless connection remains active and works properly, the wireless device must be capable enough to tackle all electrical power disturbances in IIGTF environment. So to protect the Wi-Fi equipment from physcial damage and survive in harsh industrial environment, following major points should be considered for physical protection of Wi-Fi equipment

- The input power circuitry of the Wi-Fi equipment must be galvanically isolated from rest of the circutry and antenna of Wi-Fi device in order to ensure efficient network performances despite of ground loops and HV switching problems

- The device should also be compliant with appropriate EMC/EMI standards specifically ESD, EFT and voltage surge standards as per the IEC 61000 standard series.

- As the installation of Wi-Fi devices are to be installed in indoor environment, IP30 degree of ingress protection is recommended for the $\mathrm{Wi}-\mathrm{Fi}$ device.

- During initial Wi-Fi network design, sufficient attention

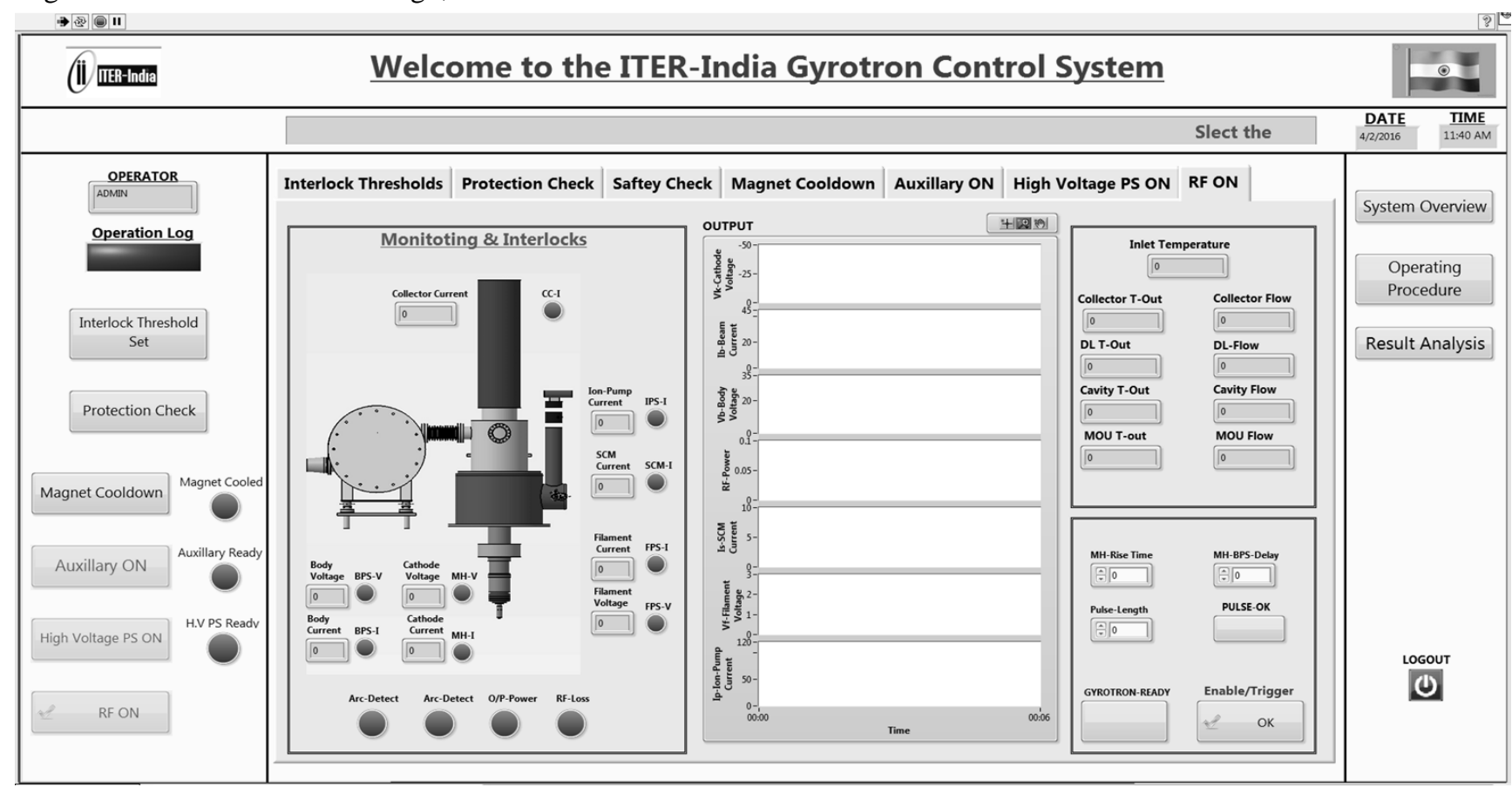

Fig.2. LabVIEW HMI for ECCS 


\section{CURRENT STATUS AND TEST RESULTS}

The software application for control of auxiliary power supplies and service has been developed in Simatic Manager using SCL language. The application consists of sequence control logic, cooling system monitoring logic, soft interlock logic. This function are implemented using different FBs and FCs in Simatic Manager. The Fig. 3 shows various programming blocks in STEP-7 software. The Fig.5 the various SCL code developed for different power supplies and auxiliary services. As the actual Test Gyrotron system is under procurement, the developed application logic has been verified using dummy test signals for all the power supplies and auxiliary services (such as cooling system). The Graphical User Interface (GUI) has been developed in LabVIEW.
This GUI communicates with Siemens PLC using NI-OPC server using NI Shared variable Engine. The Fig.4 and Fig.6 shows the programmable controllers at IIGTF. The Fig.2 shows the main GUI of ECCS while Fig.7 shows the GUI for Gyrotron Cooling manifold system where values of an existing cooling parameters (water flowrate, pressure and temperature) can be monitored. In the present architecture, communication takes place over Ethernet network. The system performance shall be tested after installation of IEEE 802.11n compliant Wi-Fi routers and clients. Initially, cooling systems instrumentation shall be first connected to Wi-Fi (Fig.8) and later other power supplies shall be connected and subsequently system performance (such as frame loss ratio, reliability and transmission latencies) shall be evaluated and test results shall be published later.

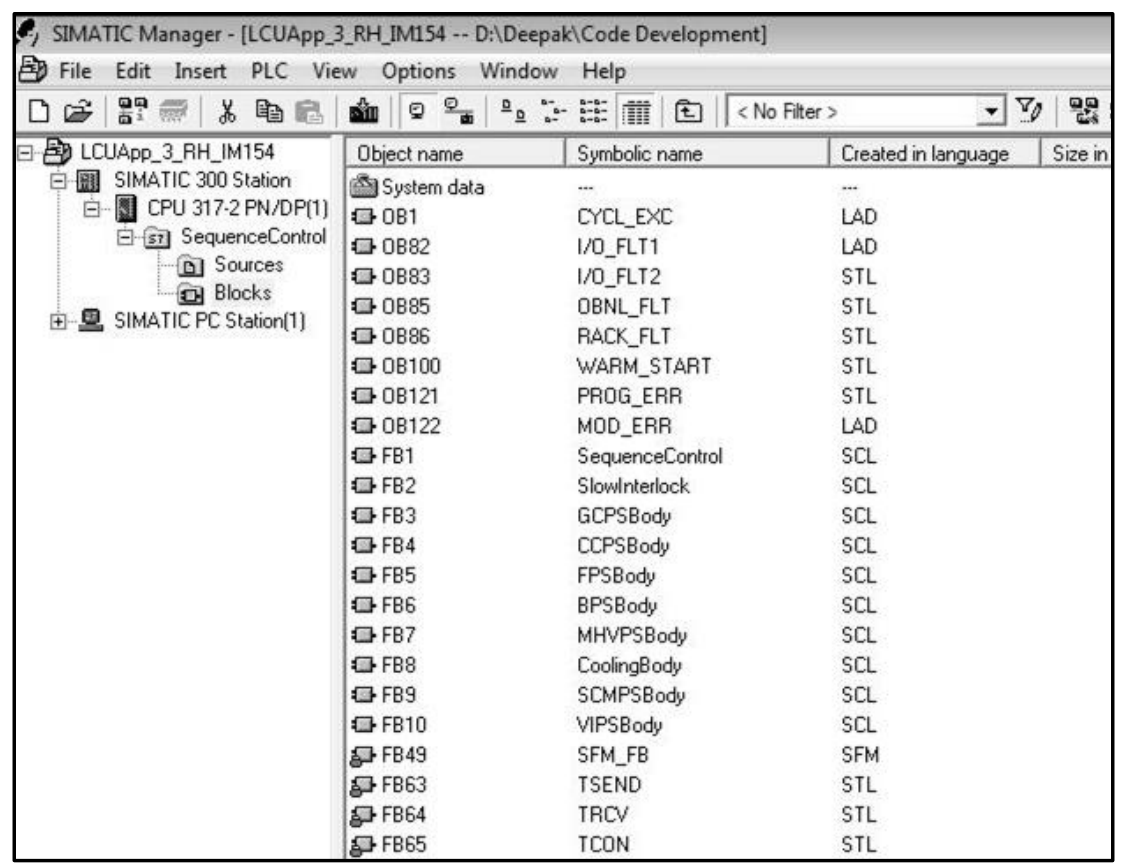

Fig.3. STEP-7 programming blocks

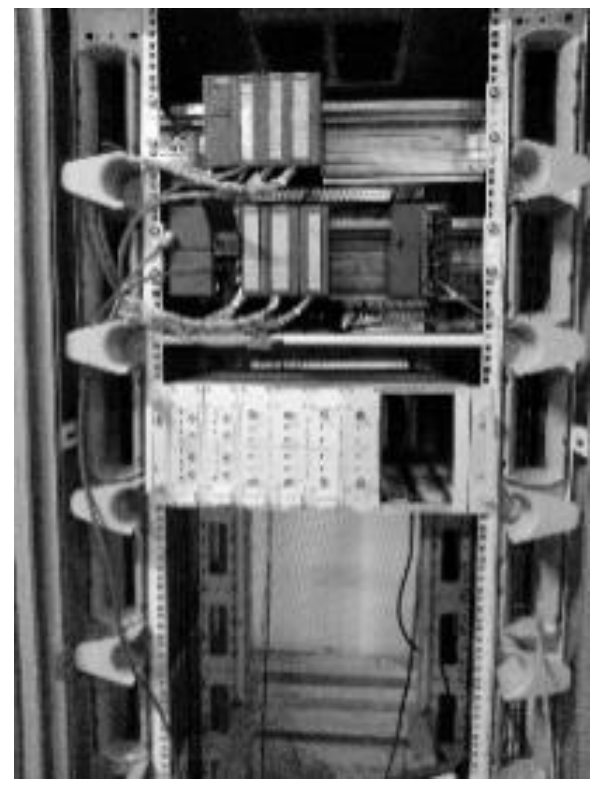

Fig.4. Siemens PLC System 


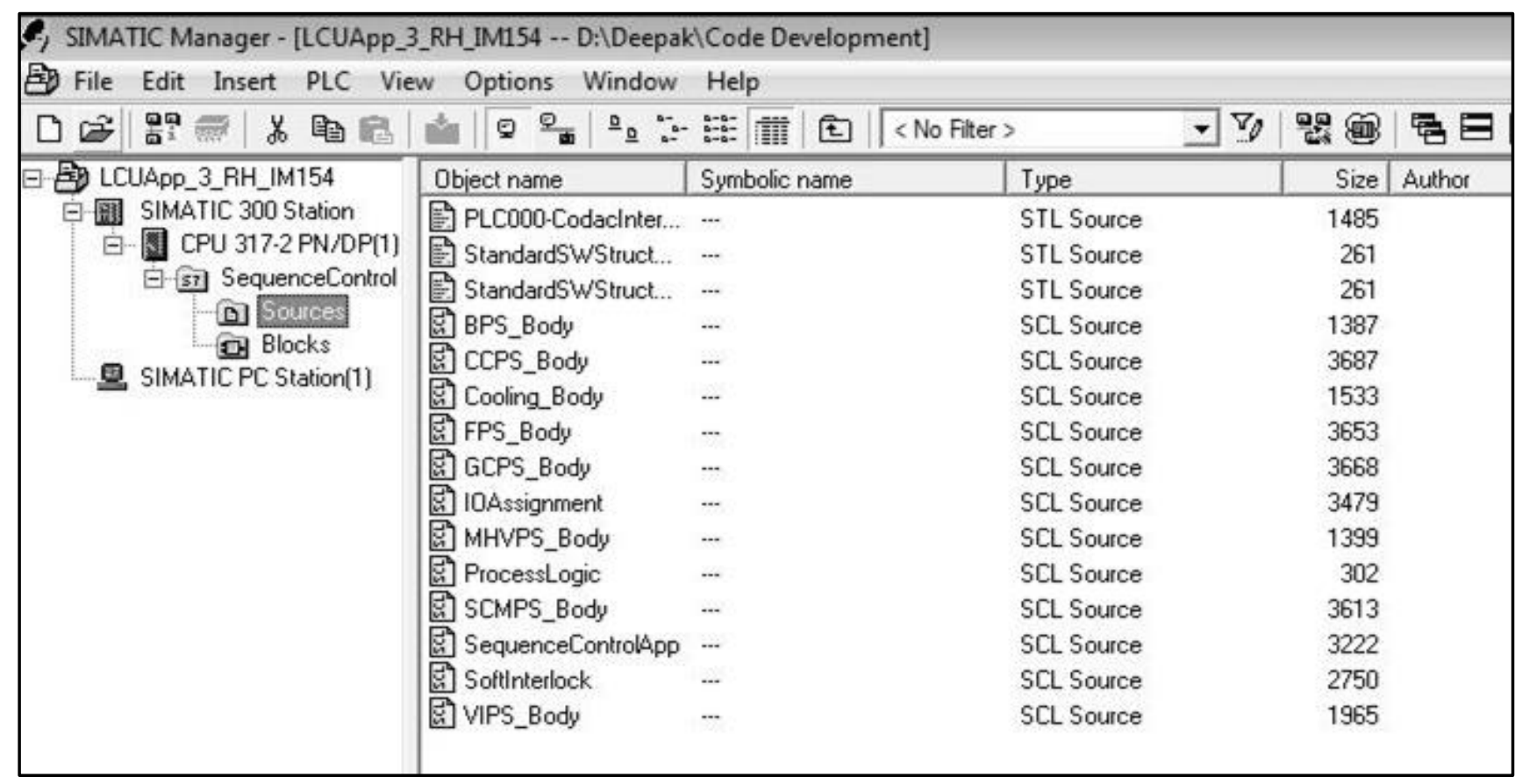

Fig.5. STEP-7 SCL source files

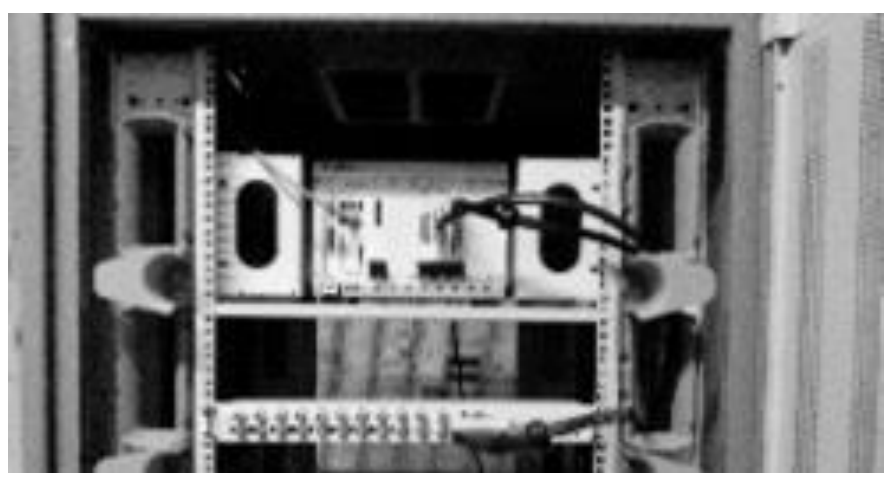

Fig.6. NI PXIe System

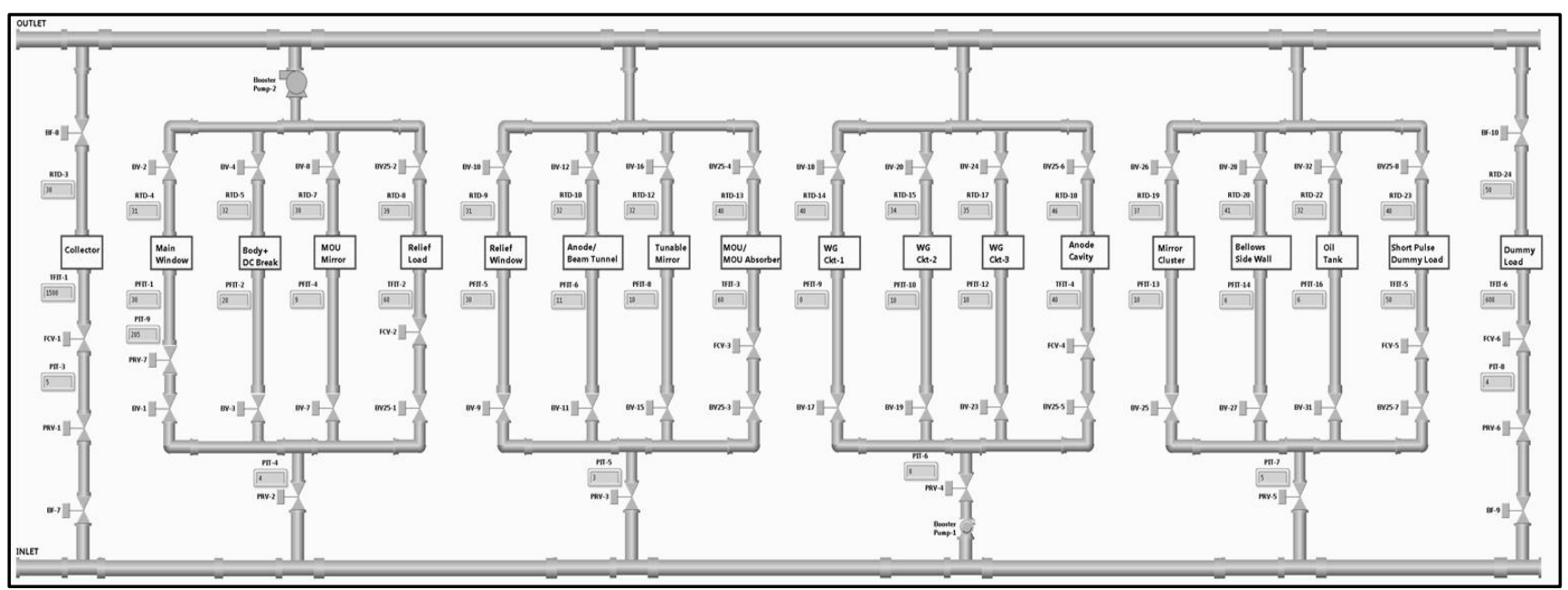

Fig.7. Cooling System HMI for ECCS 


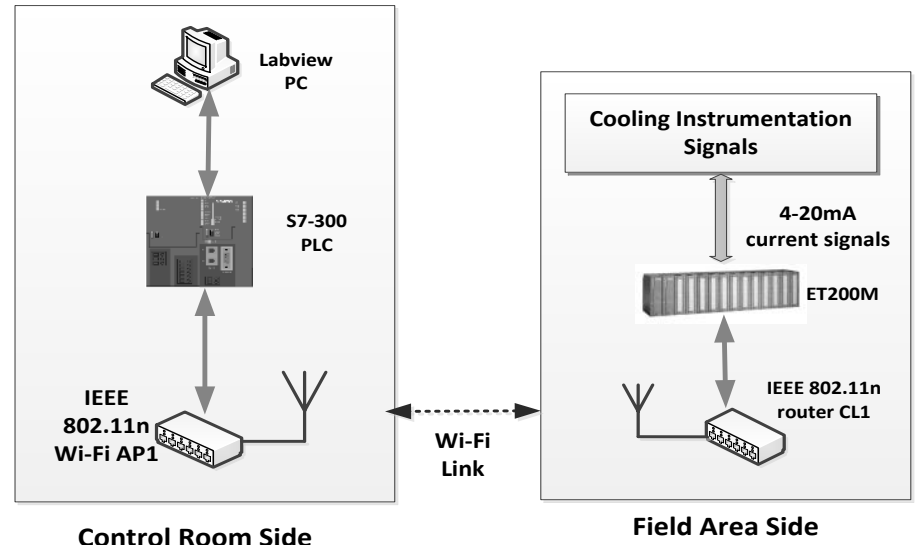

Fig.8. IEEE 802.11n Wi-Fi for Cooling System

\section{CONCLUSION}

The technology of industrial wireless comunication can be implemented for ECCS at IIGTF. In this paper, we have proposed new architecture of ECCS using wireless networks for communication requirements. A brief overview of existing ECCS architecture has been presented and a new model of ECCS architecture is proposed to reduce complexities, cost and to simplify communication requirements. IEEE $802.11 \mathrm{n} \mathrm{Wi-Fi}$ technology has been chosen for communication for ECCS because of its huge potential and high features compared to other wireless technologies. The issues regarding reliability and ecurity for Wi-Fi have been discussed and the methods to mitigate these problems are also suggested. The typical environment at IIGTF lab has been also presented and appropriate design consideration for Wi-Fi networks and Wi-Fi equipement protection to perform efficiently and reliably in IIGTF environment is also presented.

\section{REFERENCES}

[1] IEEE 802.11 Standard for Wireless LAN Medium Access Control (MAC) and Physical Layer (PHY) Specifications, Available at: http://www.di-srv.unisa.it/ vitsca/RC0809I/IEEE-802-11.pdf

[2] Federico Tramarin, Stefano Vitturi and Michele Luvisotto, "Enhancing the Real-Time Behavior of IEEE 802.11", Proceedings of IEEE World Conference on Factory Communication Systems, pp. 1-5, 2015.

[3] Federico Tramarin, Stefano Vitturi, Michele Luvisotto and Andrea Zanella, "On the Use of IEEE 802.11n for Industrial Communications", IEEE Transactions on Industrial Informatics, Vol. 3203, No. 5, pp. 1877-1886, 2015.
[4] Thomas Paul and Tokunbdo Ogunfrunmiri, "Wireless LAN Comes of Age: Understanding the IEEE 802.11n Amendment", IEEE Circuits and Systems Magazine, Vol. 8 , No. 1, pp. 28-54, 2008.

[5] Federico Tramarin, Stefano Vitturi, Michele Luvisotto and Andrea Zanella, "The IEEE 802.11n Wireless LAN for Real-Time Industrial Communication", Proceedings of IEEE World Conference on Factory Communication Systems, pp. 1-3, 2015.

[6] Chunlei Yin, Guangjun Wen and Zhengyong Feng, "Simulation Research of 802.11n Channel Model D in NS2," Proceedings of $3^{\text {rd }}$ IEEE International Conference on Computer Science and Information Technology, pp. 530533, 2010.

[7] S.L. Rao et al., "EC Power Source System for ITER", Fusion Science and Technology, Vol. 65, No. 1, pp. 129-144, 2014.

[8] Vipal Rathod, Ronak Shah, Deepak Mandge, Rajvi Parmarr and S.L. Rao, "Local Control Unit for ITER-India Gyrotron Test Facility (IIGTF)", Fusion Engineering and Design, Vol. 112, pp. 897-905, 2016.

[9] Vipal Rathod, Ronak Shah, Deepak Mandge, Rajvi Parmarr and S.L. Rao, "High Speed Analog Fiber Optical Transmission Link based on Voltage to Frequency Converter Technique for ITER-India Gyrotron Test Facility", Proceedings of International Conference on Industrial Instrumentation and Control, pp. 687-691, 2015.

[10] Profibus, Available http://www.profibus.com/technology/profibus/.

[11] Zigbee, Available at: http://www.zigbee.org/.

[12] "WIreless HART technology." [Online]. Available: http://en.hartcomm.org/hcp/tech/wihart/wireless_overview. html, Accessed on 2017.

[13] V. Abinayaa and A. Jayan, "Case Study on Comparison of Wireless Technologies in Industrial Applications", International Journal of Scientific and Research Publications, Vol. 4, No. 2, pp. 2-5, 2014.

[14] Andreas Willig, "Recent and Emerging Topics in Wireless Industrial Communications: A Selection", IEEE Transactions on Industrial Informatics, Vol. 4, No. 2, pp. 102-122, 2008.

[15] J. Song, S. Han, A.K. Mok, D. Chen, M. Lucas, M. Nixon and W. Pratt, "WirelessHART: Applying Wireless Technology in Real-Time Industrial Process Control", Proceedings of Real-Time and Embedded Technology and Applications Symposium, pp. 377-386, 2008.

[16] Tackling the Challenges of Industrial Wireless for Digital Oil Fields, Available at: http://www.moxa.com/newsletter/connection/2015/07/feat_ 02.htm 